\title{
BRECHUNG UND UMLAUT IM NORDISCHEN.
}

Die altnordische lautlehre verdient in vieler hinsicht noch eine sorgfältigere bearbeitung, als ihr seither zu teil geworlen ist, ganz besonders aber bedarf das schwierige capitel von brechung und umlaut und deren gegenseitigem verhältnis einer gründlichen untersuchung ${ }^{1}$ ), bei der namentlich die stellung der beiclen nordischen sprachzweige zu einander in betracht kommen muss. - Wenn ich im folgenden meine versuche, die lautlichen erscheinungen auf diesem gebiete aufzuklären und nach bestimmten gesichtspunkten zu ordnen, mitteile, so geschieht es keineswegs in der meinung; als wäre ich dabei immer $z u$ sicheren und unanfechtbaren ergebnissen gekommen. Vielfach bin ich nicht über das abwägen der grössern und geringern wahrscheinlichkeit fuir zwei möglichkeiten hinaus gekommen. Zwar ist mir dies vorsichtige abwägen der gründe für einander gegenüberstehende ansichten und meine zurückhaltung im urteil von gewisser seite in verwunderlichster weise zum vorwurf gemacht und es ist sogar eine ganze seite des Anzeigers f. d. alt. u. d. lit. (II, 261) nicht zu gut gewesen, ein verzeichnis solcher fälle aufzunehmen, in denen ich andern ortes meine ansicht in bescheidener form auszusprechen fll. geziemend fand - ich aber freue mich, dass ich mir in meinem urteil über wisseuschaftliche fragen die bescheidenheit bewahrt habe, die mir geziemt und auch andern jiingern leuten an-

1) I)ie beste zusammenstellung bei Blomberg, Bidrag till den germaniska omljudsläran med hufvudsakligt af́scende pă Forn-Norskan. Upsala 1s65. Den ansichten des verfassers wird man freilich in vielen fällen nicht mehr zustimmen können, so namentlich in betreff der prioritit des $i$ vor $e$ (aus $a$ ) und in betreff des $\iota$-umlautes. 
stehen wïrde. So fïllt es mir auch hier natïlich nicht ein, meine meinung irgent einem auflrängen zu wollen; vielmeh. möchte ich nur auf schwierigkeiten hinweisen und meine losungsversuche nicht vorenthalten. Wenn von berufenerer seite eine hesserc lösung gefunden werden sollte, so wïrde mich das herzlich freuen. Vielleicht bietet die folgende anspruchslose zusammenstellung fuir eine solche wenigstens cinen kleinen beitrag.

\section{1) Die brechung.}

Die sogenannte brechumg $\left.j a^{1}\right)$, charakteristisch für die nurdischen sprachen. entstand aus altem $e$ (ii) zumeist vor $r$ und $l$, besond ss mit folgendem consonanten. Die entwicklung. war folgende:

$$
\begin{aligned}
& \text { z. b. *helmr } \left.<\text { healmr }<\text { hialmr, islindisch hjallmr, }{ }^{2}\right) \\
& * h e l p a(n)<\text { healpa }<\text { hialpa, , hjálpa, } \\
& \text { *fell }<\text { feall }<\text { fiall, } \\
& \text { *herta }<\text { hearta }<\text { hiarta } \\
& \text { * ferre }<\text { fearri }<\text { fiarri; dagegen } \\
& \text { *verk }<\text { veark < verk, }
\end{aligned}
$$

weil $v j(v i$ ?) im nordischen neben einauder vermieden werden (vgl. Sievers, Jen. Liter.-ztg. 1876, art. 79, sep)-abdr. p. 11). Die neigung, in vocalischen ziveilauten den letzten vocal zu bctonen, tritt bekanntlich auch zu tage in dem 'umspringen der' quantitït' in solchen füllen wie sćı (aus sehva) $<$ sjii, triam (aus trevam), $<$ trjäm, fior (aus fidor $>$ fijvar) $<$ fjoir-, cior (aus $e(h) n r>$ ehvar) $<$ jör ${ }^{3}$ ) u. dgl. (vgl. Wimmer $\$ 20$, Sievers, Literaturztg. 1875, a. a. o. p. 11). Andere fälle der brechung s. bei Holtzmam, Altt. gr. $77-\delta()$, bei Blomberg 33. 11-44 und an andern stellen.

Der $u$-umlaut der 'brechumg' $j a$ (ia) ist nach allgemeiner

1) Ueber ia oder ja ist Pirodds traktat in snorra Edda und iiber denselben Lyngby, 'Tïlskr. f. I'hil. og Paed. II, 313; Holt\%mann, Altd. Glammatik 100; ferner Dietrich, Germania XII, 355-120; Mïbius, Germ. IX, 350 f. (auch Altuord. sprache p. 20); J. schmidı, Vocalismus II, $3: 12$ u. a. $\mathrm{zu}$ vergleichen.

2) $<=$ 'wird zu', $>=$ 'entsteht aus'.

3) Eine andere erklärung gibt Leffler, $v$-umlaut des $i$ p. 10, aum. 1, indem er andere zuriicknimmt. 


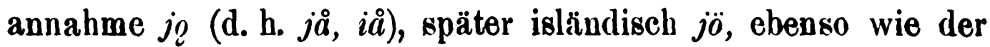
$u$-umlaut von $a$ unbestritten $g$ (d. h. $a$ ) ist, später isländisch $\ddot{\partial}$. Hier liegt es klar durch die vergleichung der verwanten sprachen, dass $a$ der ursprüngliche, $g$ der durch $u(v, o)$ modificierte laut ist, nicht aber umgekehrt $\varrho$ das urspringliche und durch folgendes $a$ zu $a$ gewandelt sein kann. Wie also niemand bestreiten kann, dass durch folgendes $u(v, o)$ einfaches $a$ zu $o$ umgelautet wird, so sollte man meinen, wäre auch unbedenklich in der verbindung $i a$ (vor $u: i g$ ) das $a$ der ursprïngliche, das $\varrho$ der gervandelte laut. ${ }^{1}$ ) Dem gegenüber hat aber Joh. Schmidt (Vocalismus II, 392 ff.) die entgegengesetzte ansicht aufgestellt, indem er sowol hierin wie in andern punkten ibereinstimmung des nordischen vocalismus mit dem angelsächsischen nachzuweisen sucht: in beiden sprachen soll $e o$ gleichzeitig aus $e$ entstanden sein durch eine art $u$-umlaut. Dies umlaut wirkende $u$ soll nun aber ein 'svarabhakti'-vocal sein, der sich zwischen $r$ oder $l$ und folgendem consonanten erzeugte, also z. b. erpa (got. airpa) < eruba< eoruba< eorbu $<$ eorb $=$ ags. eor $\delta$-; dagegen z. b. im gen. ward eorpar < earpar durch ' $a$-umlaut'. Wirklich in der angegebenen weise $z u$ erklären ist wol das io in miolkr (isl. mjölkr) $>$ *milukr, got. miluks; vgl. jedoch mialta (statt *mjalkta?), mjaltr, mjaltir (C-V.; Blomberg 41).

Wie kommt aber Schmidt zu dem irrationalen $u$ zwischen $r$ oder $l$ und folgendem consonanten? Er beruft sich auf die ältern runeninschriften - mit recht, wenn sie die neigung zur entwicklung eines irrationalen vocals erweisen sollen; mit unreoht aber, wenn sie erweisen sollen, dass dieser vocal gerade $u$ war. Die ältern runendenkmäler zeigen nümlich ganz vorwiegend $a$ in solchem falle, sehr selten $u$ oder $i, z$. b. vorahto ('I'une-stein), vulafr (dreimal Istaby-stein), vergl. afatr (Istaby - stein), gahalaiban (Tune-stcin), erilar (Varnum - stein); harivolafr (Stentofte-stein) u. a.; paraba-, arageu, falah (Björkethorp-stein). Ich brauche hier nicht mehr fälle anzufuhren als diese von Schmidt selbst beigebrachten beispiele. Andere sieh bei Bugge, Tidskr. VIII, 190. Also gerade $u$-svarabhakti anzunehmen, scheint mir nach

1) Vgl. auch Lit. Centralbl. 1875 p. 1553. 
den inschriften ganz unzulässig; eher könnte sich die brechung durch ein irrationales $a$ erklären lassen, wenn der irrationale vocal durchaus nach dem $r$ oder $l$ entstehen mulste; das ist aber nicht nötig (vgl. Lit. Centralbl. 1875, p. 1552 f.).

Die unmöglichkeit von Schmidts annahme der entstehung des ig (eo) aus $e$ durch $u$-svarabhakti ${ }^{1}$ ) wirl aber klar durch eine andere erwägung; sie passt nämlich nicht für die wörter, in denen im Isländischen (von dem Schmidt überhaupt allein ausgeht), wie $a$ und andere vocale, auch das $a$ in $i a$ verlängert ist, d. h. vor $l+k, g, p, f, m, s$, z. b. hjálmr, háls, sjálfr, hjálıa u. a. Diese verlängerung ist nicht, wie Schmidt anuimmt, eine gemeinnordische, sondern eine speciell isländische, also beiläufig ziemlich späte, da Island erst 874 besiedelt ward. Das Schwedische [und Dänische] kennt sie nicht (Rydquist IV, 28), ebensowenig das Norwegische (Aasen, Norsk gramm. § 50, anm.; Fritzner, Langes Tidskrift I, 390; Sæve, Sprogskiljaktighederna etc. Upsala 1861, 7.14; Blomberg a. a. o. p. 60; Lyngby, Tidskr. f. Phil. og Paed. II, 313); das altnorwegische homilienbuch (Gammelnorsk homiliebog, ed. Unger, Chria. 1864), welches mit längezeichen allerdings selır sparsam ist, zeigt diese doch niemals, so weit ich verfolgt habe, in den fraglichen fällen. Vou 30 fällen, die ich notierte, haben 26 ia 4 io (siolfum), stets ohne längezcichen. Reime wie fjolmeðr : hjolmum (Blomberg 60, Gislason, Aarb. 1866, p. 253 nimmt $i a: i a$ an) und andere unten p. 136 anm. 2 verzeichnete bestätigen dies. Die fraglichen $i a$ haben sich also im gemeinnordischen und bis wenigstens gegen 900 nicht von den andern schon besprochenen ia unterschieden, und sie zeigen auch in spätern Isländischen uur den einen unterschied, dass sie in der regel den $u$ umlaut nicht erleiden. Da nun hiolp (z. b. Homiliubók el. Wisén, Lund 1872, p. 30, 12; 35, 26; 39,$12 ; 61,30 ; 69,21$ u. ö.) neben hiálp, siolfum (z. b. a. i. o. 39,$4 ; 50,30 ; 52,6 ; 53, \delta$ u. ö.) neben siálfum sich ausnimmt wie nótt neben naitt, nóttum neben náttum, so pflegt man auch in hjólp, siólfum u. dgl. $u$-umlaut des langen $\dot{a}$ zu sehen;

1) die uibrigens mit der annahme in verbindung steht, dass $\dot{a}$ vor $l+$ cons. = ags. $e \dot{a}$ aus $a u$, dieses aber wider ans $a$ durch ein zwischen $l$ und conson. entwickeltes $u$ entstanden sei (Vocal. II, 402 ff.). 
man nimmit also an, die verlängerung sei vor dem wirken des flexions-u-umlautes eingetreten. Nun ist aber der flexions"-umlaut eine dem Isländischen und Norwe,rischen principiell gemeinsame erscheinung, nur dass er im Norwegischen noch - sehr oft unterbleibt. Man sieht, dass vor der besiedelung Islands das aus $a$ entstandene flexions- $\iota$ (auch ausser dem nom. sing. fem. $=$ nom. acc. pl. ntr.) schon zu wirken begonnen haben muss, ohne dass doch dieser übergang sehon ein völlig regelmässiger, gesetzmässiger newesen wäre, was er erst auf Island ward. Dem der verkehr zwischen Isiand und Norwegen, wenu er auch noch so lebhaft war, kounte höchsteus einzelne wörter und formen heriiber und hiuüber tragen; zemeinsame lautentwicklumgen so eigentümlicher art kounte das entlegene Island mit dem norwegischen mutterlande aber doch wol nicht mehr gemein haben, falls nicht eben die keime und ansätze schon in der periode der westrordischen spracheinheit vorhanden waren.

Wie ist es dann aber möglich, dass eine speciell isländische lautentwicklung wic die vocaldehnung vor $l+$ conson. vor einer gemeinsam westnordischen lauterscheinung eintrat? Das ist cben unmöglich, und daher wird man sich nach einer andern erklärung umsehen miissen; und die ist vielleicht so zu gehen 1):

Wenn der staudpunkt des Norwegischen mit seinem bald vorhandenen, bald fehlenden flexions- $\iota$-umlaute auch der des ältesten Isländischen war, wic doch anzunehmen 2), so traf der eintritt der dehnung z. b. die formen sialfum und siolfum, wie

1) Aehnlich schon Blumberg p. 60.

2) Daflir sprechen auch die reime der ältern skalden. So in der dem Bragi mugeschriebenen Ragnars-drípa (Nin. F. ed, Jinss. 14ti) heisst es in der atalhending: háls, in bols of fyllla, d. i. hals : bols (bals ist nicht nötig, da die drápa auch soust statt að̇alh. assonanz hat, was beilïufig wie manches andere metrische für ihr alter spricht). Skothending (assonanz) pflegt man gar nicht fïr die liingebestimmung heranzuziehen, doch ist auch für skothending offenl)ar die regel gleiche quantitit, wenn auch diese regel nicht von allen skalden gleich strenge beobachtet wird. Egils strophen zeigen nur wenige ausnahmen. also darf man aus tolf $\therefore$ skelfi (Egilss. 1856) s. 184, hier an stelle der adoulh.; siskelfir : sjalfan 144 in skh.; gelgju-scil a galga 116 in skh. folgern, lass im X. jahrh. auf Island die dehnung noch nicht eingetreten war. Dasselbe wird für 
das altnorwegische hımilienbuch zeigt, neben einander; und es entstauden daher die formen siálfum und siólfum (wie fillk $>$ folk). Dass die formen auf $\dot{o}$ nachher mit den $u$-umlauten eines langen $a$ zusammengeworfen wurden und daher auch deren schicksal teilten (d. h. aufgegeben wurden), das ist sehr natürlich.

Der schluss liegt nun nahe, dass, wie die in rede stehenden wörter mit $i a$ vor $l+$ consonant $\mathrm{durchweg} i a$ zcigen, auch die audern wörter mit ia (ig) vor $r$ und $l+$ conson. ursprïnglich iiberall ia hatten, dass aber, als der Hexions-ı-umlaut im Isländischen rege $1 \mathrm{~m}$ ässig eintrat, die verlängerten ii diesen unlaut nicht erlitten, wol aher die kurz gebliebenen ia, z. b.:

$$
\begin{array}{llll}
\text { hialp }(u) & <\text { hjálp, hjölp, } & & \text { hialt }(u)<\text { hjolt, } \\
\text { hialpa } & <\text { hjálpa, } & & \text { hialta }<\text { hjalta, } \\
\text { hialpom } & <\text { hjálpom (hjölpom), } & \text { hialtom }<\text { hjoltom. }
\end{array}
$$

Schmilt freilich, der die entstehung der $\dot{a}$ ganz anders erklärt, muss sich auch die entwicklung der iá ganz anders zurechtlegen. Ist seine herleitung des $i$ aus au aher an sich schon bedenklich, so wird sic erst recht unmöglich, sobald sie auf die iá angewant werten soll. Hier müste er \%. b. hiálmr so entstehen lassen: *helmar $<*$ hehumar $<*$ heolumar $<$ *heolmar $<$ healmar $<$ hjalmr. Ehe der themavocal a das co zu ca umlauten konnte, muste aber das irrationale $u$ abgefallen sein. Um aber aus healmr $<$ healumr $<$ heaulumr $<$ hiálmr nach schmidts regel entstehen zu lassen, wïrde das

das ende des 10. jahrh. tiir Norwegen wahrscheinlich durch die Vell-ekla des Einarr skálaglanı (um 999), wo wir finden (Hkr. ed. Unger 139): jalks: fylki in Skh.; es ist aber gerade in Vellekla das geset\% der slrirhen quantitait bei skothending tist ausnalumslos durehgefihrt; ferner in Vellekla: hjalm : hilmir (Hkr. 122 in skh.); folkeflandi fylkir (1:38 in skh.); bei Ilallfredr vandredaskald (der iibrigens die gleiche quantität allch ziemlich streng beobachtet) gleichzeitig für Island vielleicht dureh hjalm : hilmir Forns. 20s', 3, ebenso vielleicht in der mangelhaften aralh. skalm-öll : valdit Forns. $211^{3}, 6$, und viel früher füir Norwegen im 9. jahrh. (denn ich sehe keinen grund die iichtheit der ihm zugeschriebenen strophen im allgemeinen zu bezweifeln) bei Hornklofi hjalm-: hilmir $\mathrm{Hkr}$. to in skh. Bei Glúmr, einem Isländer, finden wir aber in der Gráfeldar llápa um 9১0 báls: Hálsi (Hkr. 134) in à̉alh. (s. Gislason, Aarb. 1866, 201 f., ulberhaupt $245 \mathrm{ff}$.). 
nochmalige auftreten und verschwinden der svarabhakti nötig sein. Dass aber, abgesehen von andern bedenken, dies zweimalige auftreten und verschwinden der svarabhakti in ein und demselben worte einen starken glauben verlangt, gibt Schmidt selbst zu und nimmt daher zur erklärung durch falsche analogie seine zuflucht. Nun ist es zwar etwas vortreffliches um die erklärung durch analogiebildungen und im Nordischen, zumal im Ostnordischen, haben sie eine grosse rolle gespielt; aber jede analogiebildung setzt doch eine imposante mehrheit der fälle, die eine minderheit beeinflusst, voraus, eine viel gebrauchte flexionsform, von der eine minder gebräuchliche beeinflusst wird oder ähnliches, wovon hier jedoch nicht die rede sein kann. Wir werden aber eine erklärung, die $a$ und iá vor $l+$ conson. gleichmässig erklärt, einer andern vorzuziehen haben, zumal die entgegenstehende ansicht Schmidts sogar bei der erklärung des einfachen $\dot{a}$ nicht unbedenklich ist.

Konnten schon die angeführten gründe die fragliche ansicht über ia und io keineswegs als wahrscheinlich erscheinen lassen, so kommt dazu doch noch ein weiteres moment gegen dieselbe, das von Schmidt nicht berlicksichtigt ist, wol aber besondere beachtung verdient. Das ist nämlich das verhalten des Schwedisch - Dänischen (Ostnordischen) zum Westnordischen in betreff des $i a$ und io (iö). Im Ostnordischen entspricht nun zunächst in einer anzahl von substantiven io und zwar durch alle casus hindurchgehend, dem mit ia wechselnden io (isländ. jö) des Westnordischen, und zwar

1) vor $r$ + consonant: a) in iorp (got. airpa), hiorp (got. hairda), *hiork $\left.{ }^{1}\right),\left(>{ }^{*}\right.$ herka [?] pl. biark-ar?), giorp (got. gairda). Diese vier sind $a$-stämme und feminina, stämme erpa-, herpa-, berka [?], gerba- durch stamm-i-umlaut (s. unten) ungelantet in hirða $>*^{*}$ herठja, hirठir $>{ }^{*}$ herठjar, birkja, birki-viðr. b) in biorn ( $>^{*}$ birnur? isl. pl. birn-ir), hiort $[e] r$ ( $>{ }^{*}$ hirtur? acc. pl. hiort-a), fior $b[e] r$ ( $>$ * firpur? pl. fir $\delta$-ir, acc. fiort-u).

1) Altschwedisch nicht belegt, neuschwedisch bjork, dänisch birk. Vgl. jedoch biork in Sv. Dipl. 18. 268 (Rydq. IV, 125). 
2) vor $l+$ couson. nur in skiold $|e| r$ ( $>$ *skildur, isl. pl. skild-ir, got. skildus), denn miolk (isl. mjölkr) gehört nicht hierher (s. oben p. 134).

3) vor einfachem $l$ : kiol (isl.kiölr, $>*$ kilur?, pl.kilir), fol (isl. fjöl, fem. 'brett, diele'. [Das adj. giol in giolskaper (Rydq.IV, 125), daneben gil, gail scheint speciell ostnord. zu sein.]

4) Vereinzelt: miok (daneben mikil), miop $[e] r$ ( $>^{*}$ mepur oder *mibur?). Da smior (isl. smjör und smör, dän. smor), wie man es auch erklären möge (s. Cl.-Vigf. 572 b) jedenfalls nicht hierher gehört' ${ }^{1}$ ), werden die wesentlichsten beispiele wol erschöpft sein (vgl. Rydq. IV, 179 und fürs Altdänische Wimmer, Navneordenes böining i ældre Dansk p. 33; Jessen, 'lidskr. V, 198) und absolute vollständigkeit ist ja hier nicht von nöten. Zunächst für unsere frage gleichgültig sind diejenigen wörter, deren stamm auf $v$ - (urspr. va-) ausgieng, die also stamm-uumlaut haben, z. b. kiot, isl. kiöt (daneben die ältere form ket), dat. kiölvi; miol (Norrb. mil), isl. miöl, dat. miölvi u. dgl. Diese sind weiter unten in anderem zusammenhange zu besprechen.

Wir werden wol tun, im weiteren verlaufe die wörter mit $l$ (und $l+$ conson) nach der brechung von denen zu scheiden, in denen die brechung dem $r+$ conson. vorhergeht. $\mathrm{Zu}-$ nächst aber legen wir uns die frage vor, ob nicht alle die genannten wörter ursprunglich io hatten, welches nur im Westnordischen vor $a$ der endung zu ea ward, so dass also diese fälle Schmidts theorie zu bestätigen geeignot wären. Es wäre danu hier das Ostnordische auf der ältern stufe stehen geblieben. Dieser auffassung stehen aber mehrere gründo entgegen :

1) erscheineu auch im Ostnordischen in diesen wörtern vereinzelte ia neben dem sonst durchgehenden io, und zwar gerade in den ältesteu quellen, so a iardu Upl. L., iarp-eghandi VGL, von dem häufigen biarn der runeninschriften (neben Biurn, Biaurn) zu schweigen. Dabei ist zu betonen, dass ausser dem nom. acc. [meist sy.] von den meisten der aufgezählten wörter in den ältern ostnordischen schriftdenkmälern wenig oder nichts belegt ist.

2) zeigt in den wörtern mit $r+$ conson. der reine, durch

') Anders jedoch Leffler, On v-omljudet af $i$ etc., Ups. 1877. 
keinc endung beeinflusste stamm westnord. durchaus ju : bjarıdír, iarb-eign, iarf-hús u. dgl. (ver.jinzelt noch Ostnordisch, wie wir sahen); Biark-ey (Altschwed. Biark-ö, d.i. Biark-ö); hjard-hundr, hjard-sveimn; hjart-skinn, hjart-kolla. Da nun die brechung schwerlich in eine so alte zeit hinaufreicht, dass damals noch der stammauslaut in zusammensetzungen erhalten zu denken wäre ${ }^{1}$ ), und noch weniger der noch jüngere (s. u.) $u$-umlaut, so zeigen $a$ - und $u$-stämme hierin keinen unterschied. Ebenso bei $l+$ conson.: skiald-maer u. dgl. - Bei einfachem $l$ aber zeigt auch das Westnordische jö (io) in kjöl-fari, kjölsýja, daneben jedoch kjal-tré, lijal-sog; von fjöl finde ich nur fjal-högg, wol aber von mjöðr: mjöठ-drekka, mjöठ-kona, mjöठrann [miök-siglandi ist natürlich mit den angeführten fällen der zusammensetzung nicht auf éine stufe zu stellen];

3) hat neben jenen rerhältnismässig wenigen fällen dlas Ostnordische in der grossen mehryahl der fälle durchweg. ia entsprechend westnordischem mit $j g$ vor $u$ wechselnden ja, z. b.:

gjof [u], gen. gjafar, gjaf-ord, gjaf-vinr u. dgl.; ostnord. giaf (daneben gif Rydq. II, 60 f.), gen. giafar etc.;

gjgld[u], gen. gjalda, gjald-dagi, gjalda (inf.); ostnord. giald, gialda etc.;

fjoll[u], gen. fjalla, fjall-skógr, fjall-slétta, fjallmaðr ete.; ostnord. fiall, fialla u. s. w.;

fjöðr, gen. fjaðrar, fjaðr-hamr, fjaðr-lauss ete; ostnorrl. fiaber, fjabrar etc.;

ja/il, dat. jofnom, fem. jofn[u], jafu-aldri, ja/i-fagr etc.; ustnord. iamn (iamn), iamnom, iamn;

und so noch in sehr zahlreichen fällen;

4) elscheint es doch mindestens auffallend, dass jene ostnordischen durchgehenden io, wenn sie urspriuglicher als in waren und durch die folge der consonanten aus $e$ (i) entstanden, sich nur in substantiven finden, in denen flexionsendungen mit " die erklärung des io aus ia (zunächst vor diesem ") nale legen; niemals aber in verb. Warum iorl, iorpur, aber niemals biorga, sondern biarga? warum skiolder, skioldar, aber

1) Jedoch noch in den ältern runendenkmälern Hlevagastir, Hapuvulafr u. a. 
nicht * giolda, sondern nur gialda? warum kiol, kiolar, aber nicht stiola, sondern stiala (sticela)? u. dgl. m.

Diese vier momente erwägend werden wir zu folgendem resultate kommen: Das im Ostnordischen in der mehrzahl der fälle durchgehende $i a$, dem im Westnordischen $j a$, vor $u$ mit jo wechselnd, entspricht, während auch der stamm im Westnordischen in zusammensetzungen $j a$ zeigt - dies $i a$ wird man in eben dieser mehrzahl der fälle dem io (jö) gegentiber unbedenklich für das ältere halten müssen, indem westnordisch $j$ o vor $u: j a$ sich verhält $=$ westnordisch $\varrho$ vor $u: a$. Das aber macht das gleiche verhältnis auch für die wenigen andern wörter wahrscheinlich, in denen im Ostnordischen io durchgeht, zumal vor $r+$ conson., wo in zusammensetzungen durchweg ia stelit, aber doch wol auch in skiolder (vgl. skjald-mer u. dgl.). Am ehesten wäre noch vor einfachem $l$ [und $p]$ in $u$-stämmen an ursprüngliches io zu denken, woruber unten mehr.

Wie erklärt sich nun bei dieser annahme das im 0stnordischen durchgehende io? Wol nur durch die annahme, dass io ursprünglich flexions- $u$-umlaut von $i a$ war, und dass dies io im Ostnordischen in alle casus (so auch Blomberg p. 66) gedrungen ist, wie eine ähnliche neigung zur uniformierung auch das spätere Norwegisch zeigt. Dann ist aber die erscheinung in den $a$-stämmen auffallend. Diese sind jedoch nur feminina, und in deren nom. so. (= nom. acc. pl. ntr.) muss der übergang des $a$ in $u$ (oder $o$, so in den ältern runeninschriften nach Wimmer, Navneord. böining i äldre Dansk 1). 47 hupro, hariso [Aarböger 1867], vgl. auch unten trjö, knjo) sebr früh eingetreten sein, inclem es gemeinuordisch schon abgefallen sein, also noch früher umlaut gewirkt haben muss, während die andern $u(0)$ aus $a$ erst ziemlich spät entstanden sein könnon (vergl. unten sjám, knjäm etc.). Dass unter den flexions- $u$-umlauten der durch obiges $u(o)$ bewirkte der älteste war, dafür sprechen auch noch andere umstïnde. 'Zunächst hat das Altnorwegische den umlaut immer in diesen fällen, wo $u$ abgefallen ist, während bei erhaltencm $u$ der umlaut bald vorhanden ist, bald fehlt (Möbius, Altn. sprache 18; hyd(1. IV, 172).') Ferner finden sich in Altschwedischen (Rydq.

1) Auch skaldenreime beweisen dies; so finden sich bei den Nor- 
II, 537; IV, 165) und Altdz̈nisethen (Wimmer a. a. a. p. 34) im nom. sing. fem, und nom. ace. pl. ntr. der starkem adjectivflexion formen wie gamosl ( $>$ "gamalu, westn. gomul), usul, armur, ukwr, ipur, wol zu beachten: nur vor $r$ oder l. Dass in diesen formen doch wol mehr als zufällige assimilation zu sehen ist, gedenke ich im folgenden darzulegen; auch die siemlich vereinzelten ${ }^{1}$ ) fälle in Tomamanurk (d. i. Danmörk), vorpr (vourbr), ubrigems auch vor $r+$ conson., und ont, isländ. önd in runeninschriften (vgl. Rydq. IV, $173 \mathrm{ff}$.) können in diesem zusammenhange angefubrt werden. Trat nun der flexions-uumlaut zuerst bei dem fraglichen, später abgefallenen $u$ auf, so könnte deshalb, wie in Tanmaurk, unterstutzt dureh $r+$ cons., dieser umlaut schon gemeinnordisch das $a$ des nom. sing. fem. [und nom. acc. pl. ntr.] getroffen haben (iarb-u<iorpu<iorb), und dann könnte das io, begünstigt durch die folgende consonanz (vgl. örn, örnar, börn, börna) auch in die andern formen gedrungen sein, wie z. b. in plur. börn, dat. börnum, gen. erst barna, dann aber auch börna (Rydq. II, 103 f., Wimmer p. 35). Dass das Ostnordische im allgemeinen und das Schwedische und Dänische im einzelnen die neigung zur einförmigkeit und zu analogiebildungen in hohem grade besitzen, werde ich noch gelegenheit haben auszufuhren. Aehnlich verhalten sich die norwegischen dialecte (Aasen, norsk gramm. § 102: bork, stong u. dgl., ferner mjöd, kjöl, björn, björk, jord, gjord, fjord, hjort).

Etwas anders steht es hingegen mit dem io vor $l$ (oder andern einfachen consonanten) in $u$-stämmen. Einmal nämlich ist das jö in isl. kjöl-fari, mjöठ-drekka u. s. w. in betracht zu

wegern bjóolfr hvinverski : skammu : glamma (Sn. E. ed. Jónss. 102, 26), langu : svangr $(103,23)$, herfangs : stangu $(103,28)$ u. a.; in Vellekla (uil 990), landum : branda (Hkr. 138), andur (statt ondur) : laudi (138) und sogar land (statt lond) : banda (116.136), ragn? (statt rogn) : magna (146) - alle in aðalhending; bei Eyvindr: skialdum (statt skioldum!) :aldri (Hkr. 112) in aðalh. Die gteiche form bei Egill: skialdum : valda (Egilss. 207) in aðalh. [Das ist für die frage nach der priorität des io oder $i a$ in skioldr nicht unwichtig.] Um 1000 reimt der Norweger Eilitr Guðrúnarson in der bórsdrápa: skammu : gammleið (Sn. E. 97, 24), vann : na nnu $(98,14)$; vargs : himintargu 98, 6; angr-: tangu 100, 30. (Bei gleichzeitigen Isländern wie Hallfreðr und Ulfr Uggason ist mir dergleichen nicht mehr aufgefallen.)

1) den stamm-umlaut abgerechnet. 
ziehen (vgl. uber den stammauslautenden vocal oben p. 140, anm). Sodann aber kann es auffallen, dass nur io westnordischem $j o$ und $j a$ vor $l$ etc. gegenuber steht, nicht aber $o$ westnordischem $g$ und $a$ unter den gleichen bedingungen, also:



während andererseits vor $r$ (und $l$ ) + consonant sich $a: g$ $=i a: i g$ verhält, z. b. in

ostnord. biörn, biörnar, westnord. bjorn, bjarnar,

ebenso ${ }_{n} \quad$ borker (neben barker);

ostnord. skiolder, skioldar, westnord. skjoldr, skjaldar, vgl. ostnord. bolker (neben balker).

Dennoch wird in anbetracht dessen, was soeben uber den umlaut durch ein aus $a$ entstandenes $u$ (o) im nom. sing. fem. gesagt ist und was bei besprechung des $u$-umlautes noch des weiteren erörtert werden wird, die annahme wenig glaublich, lass das gemeinnordische noch keinen flexionsumlaut des ursprunglichen $u$ in der $u$-declination gekannt hätte, und man wird auch hier wol die erklärung vorziehen mulssen, dass bei $a$ neben $o$ vor einfacher consonanz die ostnordische sprache zufällig den andern möglichen weg zur uniformierung gewählt hat als bei $i a$ neben io, indem in diesem falle io, in jenem aber $a$ die oberhand gewann, jedoch vor $r n, r k, r f(l k)$ das $o$ (ö): örn, börn; borker (bolker); rost (neben rast), pl. roste OGL; altdän. törf, örk (Jessen, Tidskr. V, 199, anm. 2); zweifelhafter sind nos (neben nas, Rydq. IV, 179; Jessen a. a. 0.; Blomberg 57); dän. aand, haand; boll, älter baller, isl. böllr (Rydq. III, 12; Blomberg 55), dän. logh (pl., Wimmer 36); nicht hierher gehört neuschwed. löler, denn isl. lauðr s. Aarb. 1866, 1). 263. Aehnlich wider im Norwegischen sak, takk, galt, katt neben stong, bork etc. und besonders neben mjöd, kjöl, fjöl u. s. w. (Aasen $\S 102$ ).

Demnach wtirde Schmidts theorie in den zuletzt besprochenen ausnabmefällen vicht geradezu unmöglich, fur die fâlle mit io vor $r$ (und $l$ ) + conson. im Ostnordischen aber 
höchst unwahrscheinlich ${ }^{1}$, für die weitaus tiberwiegende anzahl von wörtern mit $i a$ in Ostnordischen endlich unmöglich sein. Es bliebe also im grossen und ganzen bei der alten erklärung, dass io $u$-umlaut von $i a$ ist, wenn auch in einigen wenigen fällen bedenken in betreff der priorität des ia bestehen können.

\section{2) Der u-umlant.}

Widerholt hat mich die bisherige untersuchung schon auf den $u$-umlaut geführt, $z u$ dessen besprechung ich nunmehr ubergehe. Wie stand es mit diesem umlaute in der nordischen grundsprache?

Man pflegte das verhalten im Isländischen ohne weiteres als 'Nordisch' zu betrachten und das abweichende verhalten des Ostnordischen durch späteres zurïcktreten des umlautes zu erklären, so namentlich noch Lyngby ${ }^{2}$ ), sofern man iiberhaupt das Ostnordische heranzog. Den richtigen weg hat moines wissens nächst Petersen (Sproghistorie) zuerst Munch (Foruswensk. och Fornnorskans Språkbyggnad, Stockh. 1849, p. 28 ff.) und nach ihm Rydquist (IV, 171-190) eingeschlagen, ebenso Srve a.a.o. 7.9, indem sie den flexions- $u$-umlaut mit wenigen ausnahmen ïberhaupt furs Ostnordische leugneten, Nunch mit bestimmter scheidung des starken (stamm-) umlauts vom schwachen (flexions-) umlaut. In der tat muss man diese beiden arten

1) Leffler, $v$-umlaut $9 \mathrm{f}$. hält iiberall 'vor urspriinglichem $u$ ', d. h. wo in der $u$ - leclination $u$ in der endung hervortritt, eo fiir urspriinglich: beorn, aber doch wol nicht ea in biarnar fiir aus eo entstanden, wie Schmidt? (Vgl. Leffler 11.)

2) Tidskr. f. Phil og Paed. II, 29s f. mit ïbrigens beachtenswerten sillnden. Er lesuft sich namentlich auf die uniformierende tendenz des Ostnordischen; diese ist freilich sehr hervortretend, z. b. in der ausgleichung der drei personen im sing. der verba ausser im starken pert., der 1. plur. aller tempora und modi auf -um, der 2. pl. auf -in und vielem andern, so auch im verschwinden des beweglichen $v$ in $v a$-stimmen (Wimmer, Navn. p. 39; Rydq. IV, 139) und im ind. praes. der starken verba (s. u. $i$ - umlaut), wie auch in ter sonderentwicklung des Schwedischen und Dänischen. Dann müste $a$ iiberall die formen mit $g$ verdrängt haben, was aber doch nur mögiich war wo a nit $?$ wechselte, nicht auch wo (in folge des stamm-umlauts) durchgehends! staud (vgl. Winmer a. a. o. p. 33 ; Jessen, Tidskr. f. Phil. og Paed. V, i9s ff.). 
(les umlauts aus einander halten 1): 1) den sta mm-umlaut (auch starker oder toter umlaut genannt), bewirkt durch ein dem stamme angehöriges $u$ oder $v$, daher den stamm iiberall treffend, in allen casus- oder verbalformen [sofern nicht das umlaut wirkende $u$ vor eintritt des umlauts abgefallen ist, was namentlich im Ostnordischen häufig vorkommt]. ${ }^{2}$ )

2) den flexionsumlaut (auch schwacher oder lebender umlaut genannt), der von dem $u(o)$ einzelner casus - oder verbalformen ausgeht, und daher nur einzelne flexionsformen trifft. Hierbei muss man widerum unterscheiden, ob das $u$ (o) (ler flexionssilbe a) urspringlich oder b) aus $a$ entstanden ist. [So auch Leffler, $v$-umlaut des $i$ p. $9^{2}$ ].

Diese sehr verschiedenen fälle darf man nicht uber eins behandeln, wie das oft geschieht. Seit das sog. 'Altnordisch', d. l. Altisländisch nicht mehr als die gemeinnordische grundsprache gelten kann, sondern Ostnordisch und Westnordisch als einander gleich-geordnet erwiesen sind, muss man annehmen, dass nur die ubereinstimmungen des Westnordischen mit dem Ostnordischen (soweit zufall unwahrscheinlich ist) sicher den gemeinnordischen sprachstand ${ }^{3}$ ) darstellen, und das gilt auch vom $u$-umlaut.

Bekanntlich herscht der $u$-umlaut im Ostnordischen in viel geringerem umfange als im Westnordischen; es kommt darauf an diesen umfang genau zu begrenzen, und zwar wird es sich empfehlen, die erscheinungen des $u$-umlautes, wie sie sich im Isländischen am regelmässigsten ausgebildet finden, hier zusammenzustellen:

I. Stamm - umlaut:

$$
\begin{aligned}
a-v(u) & <o-v(u)|g=a| \\
e-v & <\ddot{o}-v \\
i-v & <y-v \\
a-v & <\dot{b}-v[\dot{b}=\text { langem } o] .
\end{aligned}
$$

1) So unterscheidet auch Blomberg (p. 53) einen st trken und schwachen $u$-umlant, von denen der letztere jiinger (p. 5i), aber doch noch gemeinnordisch (p. 58) sei.

2) Westnord. z.b. in benda > bandja statt bandvjan; häufiger im Ostnordischen (vgl. Rydq. IV, 139; Wimmer, Navn. 39).

3) In betreff dessen verweise ich in allgemeinen auf Munch in Annaler 1846, 219-253; Sæve, 'On språkskiljaktighederna i Svenska och Isländska tornskrifter', Ups. aus Univ. årskr. 1861. 
II. Flexions - umlaut:

in ableitungs- oder flexionssilben:

in stammsilben:

$$
a-u(o)<u-u(0)
$$

beides vereinigt:

$$
a-u(0)<g-u(0)
$$

$$
\begin{aligned}
& a-a-u<0-u-u . \\
& \dot{a}-u(0)<\delta-u(o),
\end{aligned}
$$

dies letztere nur unregelmässig und altertümlich.

Dass in II. der sogenannte umlaut auf die im Isländischen zum gesetz gewordene neigung zur assimilation 1) zurlickzufuhren ist, scheint mir namentlich in rïcksicht auf formen wie kolluðu aus kalladı und gomul[u] aus gamulu nicht zweifelhaft (vgl. unten p. 151). Uebrigens ist fur das aus $a$ entstandene $u$ die schreibung der ältesten isländischen hss. o (nicht $u$ ), wie auch in der ligatur zunächst, $a o$, nicht $a v$ verschlungen sind (s. unten auch sjóm, trjöm u. dgl.). Wie es aber bei II. steht, ob da uberall die assimilation durch die von Scherer ${ }^{2}$ ) und Sievers ${ }^{3}$ ) angenommene labialisierung hindurch entstanden zu denken ist ${ }^{4}$ ) - die frage darf ich hier aus dem spiele lassen [vgl. jetzt Leffler, $v$-umlaut etc. p. 5 f.].

Was nun den stamm-umlaut betrifft, so finden wir

1) den von $a<g(o, \ddot{)})$ auch in Ostnordischen in beiden in betracht kommenden fällen vertreten, wenn auch nicht so durchgehend und so regelmässig wie im Westuordischeu, nämlich

a) wo der stamm auf $v$ ausgeht:

stamm alv- urspr. alu-, isl. öl, dat. ölvi, ostn. $\ddot{j} /$.

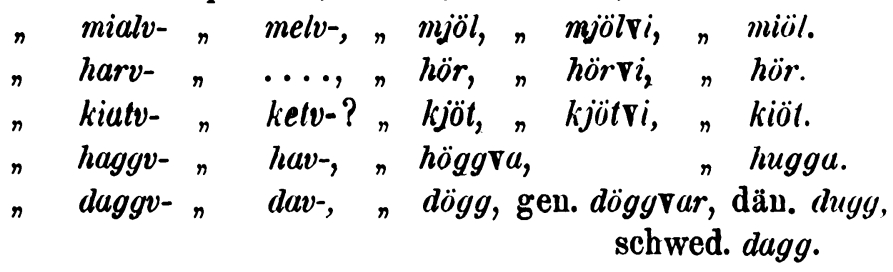

1) die sich, wie ja sonst häufig, so anch innerhalb des nordischen sprachammes im Altsehwedisehen bemerklieh macht, s. Rydq. IV, 163-7.

2) z. Gesch. d. d. Spr. 142 ff.

3) Verh. d. Leipz. Phit.-Vers. 1872, $189 \mathrm{ff}$., Lautphys. 138.

4) indem $v$ auch in die stammsilbe trat, also $a<a v$ ward, wie bekanntlich oft geschrieben; doch ist ao neben $g$ die ältere schreibung. 
stamm sparv..., isl. spörr |pl. *spörvar], dän. spurv, schwed. sparf, altschwed. spirver, dial. sparr, sporr (Rietz 654);

n arv- urspr. arhva-, isl. ör, gen. örvar, schwed. arf (Rydq. II, 61. 69);

las Ostnordische hat nämlich im interesse der gleichförmigkeit der verschiedenen flexionsformen desselben wortes das 'betregliche' $v$ auf zweierlei weise vermieden: entweder ist das $v$ als $f$ oder $v$ in alle formen getreten oder es ist uberall ausgefallen (Rydq. II, 37. 39. 69), so in

falv-, isl. fölr, acc. fölvan, ostn. fal[er], raskv-, n röskr, „ röskvan, n rasker, ebenso pranger (isl. pröngr, pröngvan) u.a.

laggv-, isl. lögg, gen. löggvar, schwed. lagg (vgl. Wimmer, Fornnord. formlära, Lund 1874, § 39, anm. 3).

sungv-, isl. söngr, dat. söngvi, schwed. säng, dăn. sang, wo das ¿ des Schwedischen doch wol noch eine nachwirkung des alten $v$ zeigt (vgl. dän. haand, schwed. havid, isl. hönd, got. handus; dän. aand, isl. önd);

b) wo ein $u$ in der ableitungssilbe steht:

Hier erscheint der umlaut im Ostnordischen nur ausnahmsweise:

havup < isl. hovio, ostn. hovop, huvnip,

iafur- $<$ iofurr, n iafur und iufur, dies nur in runoninschriften (Rydq. II, 141; IV, 171 (deutsch: ebar). Dazu etwa noch örtugh (Rydq. II, 67) [in skorvotter v-uml.]. Dagegeu in der regel kein umlaut, so in gangol, famil, hakul, stapul, axul, ankul (isl. ökkla), manlıl (isl. möttull); fjätur; [avnund = isl. öfind], harund; skallutter (isl. sköllötter), stakkutter. u. a. m. (s. Blomberg 19-29), was auf eine auch sonst nachweisliche verhältnismässig späte entstehung dieses $u$ in ableitungssilben schtiessen lässt. Darüber handelt Gr. gr. II, z. b. 103. 116 (atall, pagall, später otull, pogull u. a.), besonders aber Blomberg 19-29; Gislason, forml. p. $15 \mathrm{f}$. Zu vergleichen ist z. b. auch altschwed. apnld neben apald (isl. apaldr), hemul (isl. heimill) u. dgl.

2) $e<\ddot{o}$ (vgl. Holtzmann, Altd. gramm. I, 76 f., wo ubrigens (wol mit unrecht, wie später zu zeigen) umlaut des $v$ vor dem des $j$ bei verbindung von $v$ und $j$ angenommen wird). 
[Vgl. jetzt auch Leffler, $v$-umlaut p. $12 \mathrm{ff}$. - Die lehre von der a ufein anderfolge dieser vorgänge mag allerdings dort zum ersten male gedruckt erscheinen, ist aber sonst doch wol nicht unbekannt gewesen; ich wenigstens habe dieselbe immer schon in meinen vorlesungen in der folgenden weise vorgetragen.]

* engv-an < öngv-an;

[* melv-a? < mölva, subst. mölvir?|;

* garvjan < gerva $<$ gör $(v) a$;

akvisi < $<$ ekusi < öksi, öx (Rydq. II, 76);

got. riqiz $<{ }^{*} r \theta k v(e) r<r o ̈ k(v) r, r o ̈ k r ;$ [rekver setzt auch

Leffler an, a. a. o. p. 8];

*agviljus? < * egulir < öglir, poet. 'a kind of hawk' (..-V., wo lat. aquila verglichen wird |zu agh, angh?|;

sankvjan < $<$ sekkva $<$ sökkva, ostnord. sänkia;

sinkvan $<{ }^{*}$ sekkva $<$ sökkva, ostnord. siunka; gottl. dialect noch sinka |vgl. Leffler p. 54-59];

*stankvjan < * stekkva < stökkva, ostnord. stänkia;

stinkvan $<*$ stekkva < siökkva;

altschwed. stiunka [Leffler, $v$-umlaut p. 50-54];

* slinkvan $<{ }^{*}$ slekkva $<$ slökkva $\left.{ }^{1}\right)$;

*prangvjan < * brengva < pröngva (got. prei h $a n=d r$ in gen, ostnord. prängia, (Schmidt, Vocal. I, 53);

* angvjan < $<$ engv $(j) a<$ öngva und öngja.

*angvib $(a)<*_{\text {engub }}(u)<$ öngd, schwed. engd.

[Ueber hrökkva, ostn. rynkia; klökkva, ostn. klynka s. Leftler p. $63-67$.

Das dunkle örendi neben erindi mag hier aus dem spiele bleiben (vgl. ubrigens Schmidt, Vocal. II, 476-8). Doch gehören wol ör-r (freigebig, vgl. got. arvjô) und nökkverr, nökkurr neben nekverr, nach Bugges deutung ('l'ilskr. IX, 122-9) hierher, altschwed. auch noquar neben neqvar. - Das 0stnordische kennt diesen umlaut in $g \ddot{o r} r(v) a$, öx $(i)$, noquar; in

1) Schwed. * slekkia? (Rydq. vermutet sliuka,slykia I, 232), perf. slöck in dialecten [Leffler 13]. 
sinkva und stinkva ist dureh das unterbleiben der verwandlung des $u k<k / i$ auch ler ibergang $i<e$ nicht eingetreten 1$)$; in cngd, senkia und stenkia fehlt der umlaut, die übrigen wörter cntziehen sich der vergleichung.

3) $i<y$ (vol. Holtzmann a. a. 0. p. 74, wo aber manches zweifelhafte angefih hrt ist, z. b. frysvar; doch wol analogiebildung nach tysvar statt tvisvar, altschwed. prisvar, prysvar. [Erst nach abschluss dieser abhandlung gieng mir die kurzlich erschienene griuddliche abhandlung iiber diesen umlaut $\mathrm{zu}$ von Leffler, Om v-omljudet af $i, \overline{1}$ och ei i de nordiska språken. I, sep.-abdr. aus Ups. unir.årskr. 1877.]

ri-gg-v, isl. ryggva, altschwed. ryja, röghia [Rietz];

triggv- isl. tryggr, ostnord. trygger;

* ipvar-,$\quad$ yd(v)ar-, altschwed. ipar-; inkvar- " ykkar-, " ikar [Leffler 86-90];

Ingv- (äones) isl. lingvi, ostnord. Ing-? [Leffler 84-86];

*mirkv- isl. myrkv-an, altschwed. myrker (Rydq. II, 380), mörker; vgl. isl. neben myrkvi ein mjorkvi (C.-Vigf. 441b);

pikki- isl. bykkv-an, altschwed. piukker, vgl. auch isl. piokkr in ältern hss.;

singvan- isl. syngva, ostnord. siznga (Gottl. L. noch singia), [rol. Leffler 29-46];

*slinguan- isl. slyngva, ostnord. sliunga (gottl. dial. slinga) [vgl. Leffler 46-50];

* pringuan- isl. pryngva- [Leffler 59-60]; lingv- isl. lyngv-i, ostnord. liung 'haide' [Leffler 72-84];

*tiggv-? isl.tyggva- (Schmidt, Voc.I, 120), schwed.tugga (tiugga?).

Hierher auch siunka, stiunka und manches andere.

Langes 'y scheint hierher zu gehören in $y^{\prime} r$ (eibe) aus iv'-1, Tijr aus Tiv-l, fy(j) aus * fiv-ju²) (auch in spý-ju, snýr aus * snivir? Wimmer $[1874] \S 11 \mathrm{~d})$.

Der $u$-umlaut des $i$ ist im Ostnordischen also selten in (ier gestalt des Westnordischen als y (trygger, myrker, * ryggja?

1) Eine andere erklärung gibt Holtzmann (a. a. o. 7 i), nämlich sigqan $<$ sykkva < sökliva (eher wäre doch noch anzunehmen sinkvan $<$ synkva sökkva).

") pivan $<p i(v) a<p j a$ vgl. Fms. 1, 2\$9. Davon abgeleitet pja (st. pjaja; pjäđa) oder = pivajan? 
(kaum in prysvar; vgl. noch rynkia, $k l y n k(i) a$, wo $y$ aus $i u$ ? Leffler 65) meist als $i u, i^{1}$ ) erhalten (siunka, stiunka und stinqva, sliunga und slinga, siunga, liung, piukker); in einzelnen fällen fehlt der umlaut dem Ostnordischen gatnz, wol weil das $v$ abgefallen war, wie oft im Ostnordischen. Ob in dem iu in siunga u. dgl. wirklich die nach Scherers und Sievers' erklärung. zu erschliessenden iibergang'sstufen vorliegen (vgl. isl. piokkr, miorkvi selten und alt), das'ist nicht so sicher, wio es auf den ersten blick scheint, demn das Schwedische löst auch sonst $y$ in iu auf (vgl. kiurtil, diur; desgl. $\ddot{a}$ in ia: siang [brechung], auch siâl aus *s(al?). Dagegen sprechen übrigens auch die altertumlichsten schwedischen dialecte [vgl. jetzt Leffler]. Noch weniger ist in dieser hinsicht auf altislïndisch $e v=\ddot{o}$ gewicht zu legen (z. b. im Homilíu-bók ed. Wisén, Lund 1872: keomr 29,$24 ; 34,26 ; 36,18$ u. ö.; georle 37,13 ; meonde (= mïnde, myndi) 47, 2; eofstr 38, 3; eongum u. s. w.), denn ebenso findet sich fuir $x(=j-i)$ die auflösung $e \dot{o}(\mathrm{z}$. b. beón 28,$34 ; 29,7$ u. ö.; beóta 31, 16; feóre 32,2 ; breóbr 32, 15 u. s. w.)

4) $\dot{a}<\dot{o}$, nur in den ältesten hiss. und unregelmaissig: náungr (der nächste) < nóngr (meist lie ligatur von á für $\dot{\delta}$, auch die länge von $q$ ), z. b. Hom. 38, 9. 36 [Altnorw. hom.nang, z. b. 5, 8. 16. 17. 18]; ór statt $\dot{a} r={ }^{*} a v(i) r$ (lat. ovis) u. a. Im Ostnordischen sind mir solche altertlimlichkeiten nicht bckannt, was natiirlich nicht ausschliesst, dass sie auch da einmal vorhanden waren (vgl. Blomberg p. 59).

Fir den stamm- $\iota$-umlaut ist also gezeigt, dass dersellue sich in den drei ersten fällen auch in Ostnordischen nachweisen lïsst, wemn auch nicht so durchgehend und nicht so regelmässig wie im Westnordischen; das aber scheint sich zu erklüren einmal durch den (im Ostnord. häufigen) abfall des $v$ vor eintritt des $u$-umlauts, sodann aber durch relativ späto entstehung mancher $u$ in ableitungssilben, indem die meisten derselben zunächst wol $a, o, i$ waren. Eine principiellc vorschiedenheit rom Westnordischen \%eigt also das Ostnordische in betreff des stamm-u-umlautes nicht.

Bei dem flexions-umlaute ontsteht zunächst die frage, ob ursprüngliches $u$ (in der U-declination) schon gemein-

1) 'Umlautsbrechung' nennt das Leffler. 
nordisch unilaut gewirkt hat. Bei ia, io (hiort, bjorn etc.) war ich zu (ler ansicht gekommen (oben 1). 141), dass ia das ältere und zunïchst durch ursprïngliches $u$ in einzelnen casusformen umgelautet, dann aber $i$ in alle casusformen gedrungen sci, wie anch im fem. (ior $\%$ etc.) io aus dem nom. sing. in alle casus kam; bei kiol war die sache zweifelhaft. Bei $a$ im stamme fehlt der umlaut ostnord. durchaus, ansser in örn, börn und einigen audern vereinzelten fällen (s. oben 1. 143); also:

isländ. vondr, schwed. vander,

$$
\begin{array}{llll} 
& \text { vordr, } & & \text { varper, [vorpr] } \\
& \text { hond, } & & \text { hand, aber dän. haand, } \\
& \text { tonn, } & & \text { tand, } \\
& \text { volr, } & & \text { val u. s. w. }
\end{array}
$$

Ich grlaubte auch in diesen fällen für nom. acc. sing., dat. ace. pl. gemeinnordischen umlaut annehmen zu miissen, der aber in diesem falle iiberall dem streben nach gleichbildung zum opfer fiel (vol. oben p. 143). Diese erklärung war mir die wahrscheinlichste, weil sonst io in bjorn etc. sich schlecht, in ior/ etc. aber gar nicht erklirien liesse, und weil mancherlei ostnordische formen und namentlich gamul, annur ete. nur unter annahme vereinzelter umlaute durch das älteste der unurspritnglichen $u$ verstïndlich sind. Was nun diese formen gamul etc. (s. oben p. 142) betrifft, so haben wir uns diese wol so zu erklären: der flexions-umlaut traf zuerst die ableitungssilbe, deren voeal dem $u(o)$ völlig angeglichen ward, dann erst auch die stammsilbe, deren vocal nur zu " (später ij) assimilicrt ward;

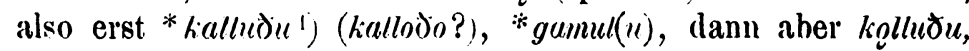
g?mul. Auf der ersten stufe stand nun, wic es scheint, das Gemeinnordische, nur dass der tibergang erst vereinzelt und unterstutzt durch dic folge gewisser consonanten ( $r$ und $l$ ) cintrat.

Wie steht es num bei unursprünglichem $u$ ? In diesem falle fehlt der unlaut dem Ostnordischen gänzlich, mit ausnahme der besprochenen fïlle vor $u$ im nom. sing. fem. und nom. ace. pl. ntr. [Der plural ligg ist nur dünisch, börn soll nach Kydı. (II, 104) vielleicht aus dem Dänischen ins Schwedische gekommen sein, aber woher hatte ihn dann das Dä-

1) So auch Leftler, $v$-umlaut 2. 
nische?] Desgleichen kennt das Ostnordische den $u$-umlaut des langen $\dot{a}$, wenigstens in den erhaltenen denkmälern, nicht, s. oben p. 150.

Die ubergänge $e-v<\ddot{o}-v$ und $i-v<y-v$ kommen schon im Westnordischen nur als stamm-umlaute vor: nicht nur in teljum, eggjum, egg (statt * eggju), sondern auch in rekkum, gefum, nemum u. dgl. (wo kein $j$ zwischen $e$ und $u$ steht) bleibt $e$ (vgl. übrigens Blomberg $18 \mathrm{f}$.). Ebenso bleibt $i$ nicht nur in liggjum u. s. f., sondern auch in bindum, digrum, digr (statt digru) u. s. w. Dass auf das von Holtzmann betonte $m \mathbf{y} k l u$ nichts zu geben ist, folgt daraus, dass $y$, wo es erscheint, in allen formen des wortes (nicht bloss vor u) erscheint, so auch im Altschwedischen (Rydq. II, 398 f.).

\section{3) Der i - umlaut.}

Auch beim $i$-umlaut muss stamm- und flexions-umlaut unterschieden werden.

I. stamm-umlaut.

$$
\begin{aligned}
& a-j(i)<e-j(i) ; \quad \dot{a}-j(i)<a-j(i) . \\
& o-j(i)<o-j(i) ; \quad \dot{o}-j(i)<x-j(i) . \\
& u-j(i)<y-j(i) ; \quad u-j(i)<y-j(i) . \\
& a u-j(i)<e y-j(i) . \\
& {[i u(i o)-j(i)<y-j(i) ; i u \text { (westnord. auch } i o)-j(i)<j-j(i) .}
\end{aligned}
$$

Fur die meisten dieser fälle bedarf es keiner belege. Dass neben $\ddot{o}$ in söfr $>{ }^{*}$ sofir, kömr $>$ komir, hnötr $>*^{*}$ hnotir u. dgl. auch $y$ als umlaut des $o$ erscheint, ist bekannt, und zwar wird sich dies $y$ schwerlich in allen füllen mit Holtzmann p. 74 auf älteres $u$ zuriuckfuhren lassen, wie freilich in synir neben sonr u. a. (vgl. ubrigens Leffler, Tidskr. n. r. II, 283, anın. 1; 284, anm. 2). Für den umlaut des iú (ii) genügt der hinweis auf glyjja (mit $j$ abgeleitet aus flin(h)an, gríta d. i. grítju (von grioit = griess) u. dgl., r. Holtzmann p. 95 f. $\quad[$ Fìj den zweifelhaften umlaut des iu (in) ist zu verweisen auf myllija $>^{*}$ miolkja? vgl. miolkr (mjölkr nur isländisch, s. oben p. 13.1, aber gen. sing. mjölkr aus * mjolkir, nicht mylkr), schwerlich auf das junge byggi, älter bjöggi]. In allen diesen fällen tritt der umlaut im Ost- und Westnordischen gleichmässig auf. Diese umlaute treten aber ein: 1) bewirkt durch ein den stamm 
endendes $j$ und zwar a) im schwachen verb. I: telja, drem $(j) a$; h) im praesensstamm etlicher starker verba: skepja; hlceja, deyja; c) in ia-stämmen aller drei geschlechter: hirð $(j) a r$, niojar, heì)(j)ar, benjar, klceð(j)a (gen.), kynja, hylli, ergi, urspr. ja(n), got. ei(n); auch im adj. sekr, sekjum, schwed. aber saker ohne 'bewegliches $j$ ', wie dieses iberhaupt im Ostnordischen oft ausgefallen ist (öar = eyjar u. dgl.; vgl. das über das entsprechende $v$ gesagte oben p. 147). Der ausfall des $j$ nach langer stammsilbe, ansser nach guttural und langem vocal (hloja, eyjar, meyjar), ist gemeinnordisch, doch, wie wir hier sehen, jünger als der flexions- $i$-umlaut. 2) umlaut bewirkt durch $i$ in der ableitungssilbe, so a) im comparativ und superlativ: $-(i) r i,-(i) s t r=$ got. -iza, -ists (yngri, yngstr); b) in -ing: Islend-ingr, hyrn-ingr, spek-ingr, erf-ingi, roen-ingr u. dgl., aber Hund-ingr [statt Hundungr? vgl. Gr. gr. II (ed. Scherer) p. 333]; bandingi statt * bandengja u. dgl. Leffler, i-uml. 14-18.

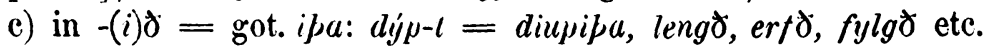
Ueber -(i)sk- u. a. s. Leffler, Tidskr. - Endlich ist noch der eigentiumliche guttural-umlaut (tekirn, dreginn, sleginn; auch degi [lengi?], genginn, fenginn gehören vielleicht hierher) zu erwähnen; dieser ist speciell westnordisch (Rydq. IV, 14; Lyngby, Udsagns - ordenes böining i jyske lov p. 20: taken, draghcel. $\mathrm{Zu}$ vergleichen noch Winmer, Navn. 53, anm.). Im ribrigen ist der $i$-umlaut in allen angefuhrten fällen gemeinnordisch.

\section{Flexions - umlaut.}

Die hier in betracht kommenden umlautungen sind dieselben wie unter I. Auch hier ist ein umlaut durch unursprilingliches $i$ zu unterscheiden.

Zunächst urspritingliches $i$ oder $j$ (d. h. $i$ oder $j$ in der gotisch-nordischen, also ostgermanischen zeit) Lat umlaut gecwirkt 1) in der 1 -declination im dat. sing. nom. [und acc.] plur.: $s y n c-i$, synt-ir $[s ! n t-i$ neben $s u n-u] . \quad$ s!yn-ir ist = got. sunjus, ace. pl. syni ist eine späte nebenform, offenbar in anlehnung. an den nom. pl. nach analogie der andern declinationen entstanden. Dass im dat. sing. das Altschwerlische selten unlaut zeigt, ist sehr beachtenswert; ol man aber berechtigt ist, daraus auf eine nebenform $-u>u u$ (got. sinuau) neben $i>j u$ (vgl. simjus) zu schliessen, ist eine frage, die in dic formen- 
lehre gehört; jodenfalls bleibt bei der gleichbildnerischen tendenz des Ostnordischen die möglichkeit, dass der vereinzelte umlaut im dat. sing. später aufgegeben ist. Dafür köunten die altdünischen formen walla (vgl. das vereinzelte altschwell. valli [Rydq. II, 145-148] im ailtern VGL) und aitd. wcendi (Wimmer p. 75) sprechen, und von diesem gesichtspunkte aus erscheint auch das vereinzelte skildi in sk. 1. 14, 5 als mehr denn ein schreibfehler, und wäre dann in mehrfacher hinsicht beachtenswert. 2) in der consonantischen declination, im nom. acc. pl. Lim gen. sing. ncetr, kýr u. a.; im Ostnordischen fehlen die belege, dagegen findet sich koa(r), soar, nattar Rydq. I, 176. 158], isländ. natr, boekr, kiyr, mýss, yeess u. a. = schwed. ncetler, böker, köör(kyr), mys. gces. u. a. [s. Lyngby, Tidskr. VI, 38 ff].

Unursprtingliches $i(\%)$. Es kommen hier zwei fälle in betracht, in denen aber noch zu prufen ist, ob das $i$ wirklich unursprünglich ist; in beiden fällen kennt das Ostnordische den umlaut nicht.

1) im sing. ind. praes. der starken conjugation. Zu neiner freude sehe ich, dass meine hier entwickelte ansicht in der hauptsache mit Leffler, $i$-uml. in 'Tidskr. n. r. II. 10-12 [vgl. auch Blomberg 70] ibereinstimmt:

$\begin{array}{llll}\text { got. } & \text { isl. } & \text { altschwed. } & \text { al thoch d. } \\ \text { far-a } & \text { fer(i) } & \text { [far(er)] } & \text { far-u } \\ \text { far-is } & \text { fer(i)r } & \text { far(er) } & \text { fer-is } \\ \text { tar-ip } & \text { [fer(i)r] } & \text { [far(er)] } & \text { fer-it } \\ \text { far-am } & \text { fọr-om } & \text { far-om } & \text { far-am(ês) } \\ \text { far-ip } & \text { far-ed } & \text { far-en } & \text { fir-at, -et } \\ \text { far-and } & \text { far-a } & \text { far-a. } & \text { far-ant, -ent. }\end{array}$

1)as zunïehst auffallende fehlen des umlautes in der 2. pers. plur. im Istiudischen erveist sich durch die vergleichung des IIochdeutschen und der andern westgermanischen sprachen als eine nicht speciell nordische erscheinung und darf daher hier fuglich unerörtert bleiben. Aber auch der singular sollte in bezug auf den umlaut sich den westgermanischen sprachen analog verhalten; wir sollten also $\operatorname{jar}(e), \operatorname{jer}(i) r$, $j e r(i) r$ erwarten. Nach iler gewöhnlichen ammahme (z. b. Sieve a. a. 0. 7; Möbius, Altn. 11) soll das fehlen des umlautes im Ostnordischen das urspriinglichere, der umlaut im Westnordischen also ein spiterer, speciell westnordischer durch nicht ursprüng- 
liches $i$ bewirkter sein. Aber nichts kann uns veranlassen eine andere nordische grundform anzusetzen als far-is, clenn ferr $>$ farir entspricht genau dem yngri $>{ }^{*}$ jungiza; dýml

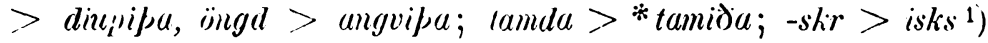
u. dgl. (dagegen 2. pl. fario, got. far-ip; uber talidir u. dgrl. s. unten p. 161 anm.). Die verschiedenheit zwischen Ostnord. und Westnord. in diesem punkte erklärt sich also wol durch eine in verschiedener richtung vorgenommene form-angleichung:

$\begin{array}{ccc}\text { gemeinuord. } & \text { westnord. } & \text { ostnord. } \\ \text { far-e } & \text { fer-i } & \text { "far-e } \\ \text { fer-ir } & \text { fer-ir } & \text { far-er } \\ \text { [fer-ir] } & \text { fer-ir } & \text { far-er. }\end{array}$

Also im Westnorl. selhloss sich die 1. sing. im umlaut an die 2. und 3. sing., im Ostnord. schloss sich die 2. und 3. sing. an die 1. sing. und den plural ohne umlaut. Darnach Ostnord. mit weiterer angleichung auch 1. sing. far-er. Altschwedische formen wie gicelder (Rydl. I, 196), stialpir (I, 199) und bicergher $(1,20 \pi)$, Altdän. gialder, hiulpeer (jyske l., Lyngby 16) darf man bei der im Ostnordischen so stark hervortretenden neigung zur formangleichung nicht gegen meine auffassung geltend machen.

2) im conj. perfecti:

\begin{tabular}{|c|c|c|c|}
\hline $\begin{array}{l}\text { got. } \\
\text { for-jau }\end{array}$ & $\begin{array}{l}\text { is } 1 . \\
\text { forr-a }\end{array}$ & $\begin{array}{l}\text { altsch wed. } \\
\text { [tor-i] }\end{array}$ & $\begin{array}{l}\text { urnord. } \\
\left.\text { fôr-(j)a } a^{2}\right)\end{array}$ \\
\hline for-eis & forl-ir & [for-i] & fôr-îr \\
\hline for $-i$ & foer-i & for $-\mathrm{i}$ & tôr-(-1 ${ }^{3}$ ) \\
\hline for-cima & frer-im & [for-olu] & fôr-îm \\
\hline tor-eip & torer-io & for-in & fôl-1̂̀ \\
\hline for-eina & firer-i & for-i(n) & fồr-în \\
\hline
\end{tabular}

Es ist wol nicht ycichgailtig, dass dem $i$ des Nordischon in Gotischen ei entsplicht, ebenso im nom. pl. der $i$-declination

1) mit umlaut nach langer stammsillse -lendskr, malskr, ohne unlaut nich kurzer liorskir, valshr, vaskr. danskr, dulskr etc. Beispiele bei Leffler, $i$-munlaut 'Tillskr. Is.

2) $j$ fiill in Nord. nach lit uger stammsilbe (ausser nach guttural orler vocal) immer atts. IIier aber anch nach $\mathrm{k} u \mathrm{r} \% \mathrm{cr}$ : gripa, skiytu.

$\left.{ }^{3}\right)$ got. $i=$ nord. $i$ in der endung scheint immer nicht dic ostgermanische lautstule, sondern eine jïngere speciell gutische entwicklung darzustellen: ostgerm. $i$ in endung und ableitungssilbe fällt in Nord. aus, s. oben und 1. 1:3.;. 
staōir > stapeis, ferner nemandi > nimandei (dem gegeniiber kann srekir $>$ sokeis analogiebildung sein statt sókir). In unserm falle sollten wir urnordisch in der 1. sing. umlaut erwarten: $f a r-(j) a$, for $-i r$ u. s. w. Weniger wahrscheinlichkeit scheint mir die annahme fuir sich zu haben, dass der umlaut im conj. perf. gemeinnordisch vorhanden gewesen, im Ostnord. aber aufgegeben sei.

\section{4) Zur chronologie der altnordischen lautgeschichte.}

Es ist gewis nicht gleichgältig fiur die erklärung lautlicher erscheinungen, in welcher reihenfolge gewisse lautiibergänge statt hatten. Für umlaut und brechung und einige damit in zusammenhang stehendo lauterscheinungen soll im folgenden versucht werden die reihenfolge festzustellen, soweit dies möglich ist.

Wann trat die brechung ein? Bugge, Hamðismál in Z. f. d. Phil. VII, 394 (zu Erpr neben jarpr) meint, dass dies 'wol im 8. jahrhundert geschah'. Der Varnum-stein und die inschrift auf einem schlangenbilde aus dem Lindholm-mor (Wimmer, Navn. 45) bieten er(i)lar $=$ jarl $(a) r$, in den jüngern runendenkmälern finden sich gebrochene und ungebrochene formen. Auf das Necrologium Augiense 1) wird man nur mit grösster vorsicht einen schluss bauen dürfen, abgeseheu davon, dass sich fiir eine zeitbestimmung nicht viel daraus gewinnen lässt. Da die hs. aus dem X./XI. jahrhundert ist, während die niederschrift der namen im original des nekrologiums doch zum teil schon frtiher stattfand, wird man isländische christliche pilger gewis nur wenige darin erwarten durfen, vielleicht nur da, wo es ausdrticklich bemerkt ist (s. 168). Uebrigens sind die namen anscheinend nicht einmal alle skandinavisch, sondern zum teil auch ags. - In betreff der nordischen namen ist anzumerken, dass zum teil der $v$-anlaut vor u, o erhalten ist, z. b. volar Anz. 99, 16 v. o., doch ulaph 98, 16 v. u., vlua (d. i. ulva?) 99, 16 v. 0.; ferner findet sich Sorli 99, z. 6, islïnd. Sörli $>$ Sarula. Die brechung betreffend

1) Mone im Auz. f. kunde der t. vol'zeit IV (1835) 19 fit. und !s fti. J. Grimm in Antiq. 'Tidski. 1843-j, p. 6i- 75. 
ist hervorzuheben, dass sich $\mathrm{nur}$ ungebrochene formen finden (so durchweg -birn, -pirn, -pern, iburbern = iofurbiorn, Anz. 99,13 v. u.), was doch nicht gleichgültig ist.

Dass sich die brachung in einzelnen fällen im Ostnordischen oder Westuordischen allein findet, spricht dafür, dass sie zur zeit der nordischen sprachspaltung noch in kraft war und demnach wol nicht so gar lange vorher begonnen haben wird (so auch Leffler, $i$-umlaut 71, anm.). Westnord. nur giof, ostnord. gif und giaf; miöl, ostn. miöl und dial. mil Rydq. IV, 125; ikul (nur in runeninschriften, s. Rydq. IV, 124), westnord. iokull, vielleicht ${ }^{1}$ ) sjau > siav-u(n) (ags. seofon, got. sibun, vgl. giof $=$ got. gibu, iafn $=$ got. ibns $)$, ostnord. $\operatorname{siv} u(n)<\operatorname{si}(v) u$ $<$ sju (schwed.) und siuv? < syv (dän.). Andererseits ostnorl. iak (dial. ik), westnord. ek; fixt, westnord. fet; sticela, westnord. stela; miata, westnord. meta, aber mjotudr; siceng neben sang, westnorl. sceing; siax, sicette, westnord. sex, setti u. dgl. (Munch p. 28).

In einer andern brechung, nämlich des langen $a$ in $i a ́$, stimmen beide sprachgruppen ebenfalls uberein. Die formen saer, sjär, sjör; sncer, snjär, snjór ; mar, mjär, mjór; slcer, sljär, sljór und andere sind wenigstens nach meiner meinung nicht anders zu erklären, als dass $a(v)$ die älteste form ist $=$ got. aiv (wie fraev- und frjov- = fraiv, aev- = aiv-); gebrochen zu $i a ̀(v)$, ursprtinglich eáv?, wie kurzes $e$ zu ia (ea); dann umgelautet durch $v \mathrm{zu} i \dot{v} v$ : scev-ar, siáv-ar, sióv-ar u. dgl. Die erklärung wenigstens von Gislason ${ }^{2}$ ), der selbst Lyngby sich noch anschliesst ${ }^{3}$ ), dass $i a ́$ aus got. ái 'durch umsetzung' entstanden sei, wird man lieutigen tages doch nicht mehr vorbringen dürfen. Got. ái ward 1) < ei, ei durch eine art umlaut (rgl. mhd. ou). Dieser ubergang ist aber zunächst nur westnordisch; die altertumlichsten schwedischen dialecte zeigen noch durchaus $a i^{\dagger}$ ) (was gegen die sonst wahrscheinliche

1) wie schon Munch, Fornsv. och. Fornnorsk. p. 24.

$\Rightarrow$ Oldn. forml. p. s9.

3) Tidskr. f. Phil. og Paed. II, 303 f. - Anders Munch a. a. o. p. 24: "snaivr $<{ }^{*}$ sneior $<[$ sncior? $<]$ snjor. Wie erklärt sich dann aler sniovar?

i) So ausser den altertiiulichsten dialecten des schwedischen hauptlandes im Gottl. L. und Gottl. dial. und in den tinuisch-schwedischen 
erklärung spricht, dass $i$ vor $v$ verklang, daher aeiv $<(e v)$. Got. $a i$ ward aber 2) $<\dot{a}$ aus $\dot{a} i$, so namentlich vor $r$ und $h$ (árr, für $=$ faihs $=\pi \circ \iota x-\iota \lambda-o s$, sár, $\dot{a}, \dot{a t t a}=$ aihta u. a.

Darf man aus allem angefuhrten einen schluss ziehen, so ist es der, dass die brechung gemeinnordisch ist, aber nicht sehr lange vor der sprachspaltung eintrat. Erwägen wir nun das chronologische verhältnis der brechung zu den umlauten, zunächst zum $i$-umlaut. - Wo stammhafter $i$-umlaut $\mathrm{zu}$ erwarten ist und ausserdem in den sechs masculinen der $u$-declination erscheint neben $i a($ ig) ein $i$, wo Hexionsumlaut zu erwarten ist ein $e$, nämlich im sing. ind. praes.; z. b.

girn- (gern-), giarna, girnast $>$ "gernjask;

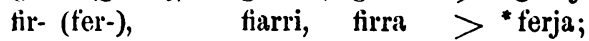

skild- (skeld- ?), skiald-, skildir $>{ }^{*}$ "skildjur, skildir $>{ }^{*}$ "skildjar

birn- (bern-?), biarn-, birnir $>$ •birnjur; nom. sing. m.;

birg- (berg-), biarga, birgja $>$ bergja, Birgir;

hilp- (help-), hiálpa, hilpir (Norges gamle love II, 43) > helpjar; Die 2. sing. praes. aber lautet bergr, helpr $>{ }^{*}$ bergir, *helpir.

Man könnte zunächst zu der annahme kommen, die verschiedenheit erkläre sich dadurch, dass in den einen fällen $i$, in den andern $e$ der stammvocal sei, und in der tat wird uran wol für die $u$-stämme im Ostgermanischen $i$ als stammvocal annehmen müssen: hirtu-, firðu-, skildu-, birnu-, miðu-, kilu-.') Denn dass in skildir ( $>*^{*}$ skeldjur), synir $(>$ sunjur) $;$ wie in firra (statt ferja), bergja (statt bargja), in bergr, helpr aber $i$ den umlaut wirkt, kann das verschiedene verhalten doch nicht begriinden. In den andern fallen aber spricht gegen die annahme eines $i$ im stamme, abgesehen von der vergleichung der westgrermanischen sprachen, folgende zusammenstellung:

berg- biarga, bergr-, birgja > bergja;
geld- gialda, geldr, gildi > "gildja;
help- hjálpa, helpr, hilpir > lelpjar;
helm- hjálmr, - , hilmir > helmjar;
gern- giarna, - , girnast > gernjask.

dialecten (Rydq. IV, 141). Dies ai verhält sich zu islïnd. $c i=a y: \iota y$. Auf ay geht auch das ai (ei) der esthnischen Inselschweden zurllck, z. b. in $a i, e i$ insel; aik, eik = isl. eykr; raik, reik = isl. reyjlir (Ruszwurm, Ueber die sprache der Inselschwedeu, Reval 15505 ).

1) Anders Leffler, der bern- ete. als nordische grundform ansieht; s. jedoch uuten p. 159 und 162 anm. 1. 
Man sieht daraus, dass nicht im stammvocal hier der grund zur abweichung liegt. Dann aber kann diese nur daraus sich erklären, dass einmal flexions-umlaut, das andere mal stamm-umlaut vorliegt, indem beide unlaute $\mathrm{zu}$ verschiedener zeit eintraten, so dass der stamm-umlaut noch auf ungebrochenes $e$, der flexions-umlaut aber schon auf gebrochenes $e$, also $e^{a}$ einwirkte (in welchem falle das $a$ wider verklang) oder die brechung iiberhaupt verhinderte. hjálpa, helpr zeigt ausserdem, dass die dehnung des ia (ea) nicht nur nach dem stamm$i$-umlaut, sondern auch nach dem flexions-i-umlaut eintrat. Also können wir folgende entwickelungsstufen ansetzen:

$\begin{array}{lllll}\text { 1. } & 2 . & 3 . & 4 & \mathbf{3} .\end{array}$

*helpa, helpa, healpa, hjalpa, hjálpa;

- helpir, helpir, [healpir, helpir,] helpr;

*helpjar, hilpir;

* bargja, bergja;

* skildjur, skildir.

Demnach läge der stamm-i-umlaut vor, der flexions$i$-umlaut nach oder gleichzeitig mit der brechung. Leffler scheidet hier nicht den stanm-umlaut rom flexions-umlaut, er verlegt beide vor den cintritt der brechung ( $i$-uml., Tidskr. 247); daher muss er formen wie helpr, lergr anders erklären als ich, nämlich durch analogiebildung: wie drep, drepr statt *drepe,"*dripir (= Ostuord. s. oben p. 155), so auch helpr, bergr statt *hilpir, birgir. Hier bleibt aber die grosse schwierigkeit, dic Leffler p. 11 f. nicht genulgend löst, dass die 1. sg. hier nicht *helpe, ${ }^{*}$ berge, sondern healje, bearge geheissen haben muss, woraus wol die ostnord. aualogiebildungen *hialper, gialder, nicht aber westnord. helpr, geldr sich erklälen lassen. Auf die ostnordischen formen giaelder ti. dgl. (s. oben p. 155), skioler u. s. w. darf man ubrigens aus dem dort augefuhrten grunde nicht $\mathrm{zu}$ viel gewicht legen. Da derselbe umlaut gleichzeitig 1) bargja < beryja und bergja < birgja umgestaltete, kounte er jenes erstere bergja nicht noch weiter in lirgja wandelu.

1) Leffler, der diesen $i$-umlaut (Bidrag til liran om $\mathrm{i}$-omljudet in 'Tidskr. n. r. II, 1-19. 146-180. $231-320$ sehr eingehend behandelt, hält ihn fïir :ilter als die andern $i$-lunlaute (15i. 246), nïulich für gemeingermanisch (266), was mir in dem angenommenen untange, zum wenigsten für den flexions-umlaut, nicht wahrscheinlich werden will. 
Kann das hier gewonnene ergebnis auch nicht als ein völlig sicheres bezeichnet werden, so ergibt sich dagegen fïrr den stamm-u-umlaut zweifellos, lass derselbe nach der brechung eintrat, denn er wirkt schon auf den gebrochenen vocal, z. b. got.hairus < hjorv- (vgl. alu- < glv-, hauh- < háu-, $<$ háv-, aber ostnord. haugh-, högh-, vgl. isl. haugr), got.fairhenes $<$ fjorv- u. s. w.

Das verhältnis des $u$-umlautes zum $i$-umlaute darf man nun nicht ohne weiteres so zusammenfassen, dass der $i$-umlaut im Gemeinnordischen völlig entwickelt, der u-umlaut aber noch in der entwicklung begriffen wäre, mithin der umlaut durch $u$ viel jünger sei als der umlaut durch $i$. So einfach liegt die sache doch nicht. Der flexions-umlaut durch unursprüngliches $u$ ist allerdings im Westnordischen ziemlich allgemein entwickelt, zeigt sich dagegen im Ostnordischen nur in bestimmten fällen in geringen ansätzen. Aber auch für unursprtingliches $i$ ist nirgends mit sicherheit ein umlaut als gemeinnordisch nachzuweisen. Bei ursprtinglichem $i$ ist der umlaut gemeinnordisch, aber wahrscheinlich auch bei ursprünglichem $u$, wenn das auch nicht völlig sicher ist (vgl. oben p. 143. 151). In betreff des stamm-umlautes ist gleichfalls sowol bei $i, j$ als auch bei $u, v$ ein grundsätzlicher unterschied zwischen Ost- und Westnordisch nicht zu constatieren; und aus dem weniger regelmässigen auftreten dieses umlautes im Ostnordischen darf ein späteres eintreten des stamm- $\iota$-umlautes als des stamm- $i$-umlautes mit sicherheit nicht gefolgert werden.1) Für die priorität des $i$-umlautes spricht allerdings das oben wahrscheinlich gemachte verhältnis beider umlaute zur brechung; vgl. auch unten $v j$ in *garvja u.dgl. p. 162.

Fur den flexions-umlaut wird die priorität des $i$-umlautes dadurch wahrscheinlich, dass er in seinem vorhandensein und fehlen sich ost- und westnordisch ziemlich genau in denselben gränzen bält, während der $u$-umlaut nur in wenigen, und zwar den am frubhesten eingetretenen fällen gemeinnordisch ist. Die wirkung des erstern muss also zur zeit der sprachspaltung

1) Got. fairguni < fearguni < feorguni < isl.fjorgyn künnte dagegen angefiihrt werden, kann aber allein doch nichts beweisen. Vgl. Blomberg 23. [Leffler sieht in dem eo hier 'umlautsbrechung'. ebenso in Mjolnir, Fjolnir, v-uml. p. 10.] 
im wesentlichen vortiber gewesen sein; nicht so die des uumlautes.

Was beweist der sogenannte rückumlaut, der der regel nach nur nach kurzer stammsilbe eintritt? Natürlich lässt dieser sich nur so erklären, dass der umlaut in den betreffenden fällen nicht etwa wider fortgefallen ist (warum danm nicht auch in tel, telr, ben statt * benja u. dgl.), sondern ülerhaupt nie vorhanden war. ${ }^{1}$ ) Was beweist nun also

$\begin{array}{ll}\text { ketil-l } & \text { telja } \\ \text { katl-i, st. }{ }^{*} \text { katili } & \text { talda, st. }{ }^{*} \text { taliòa } \\ \text { kọtl-om, st. }{ }^{*} \text { katilam } & \text { tọldum, st. }{ }^{*} \text { talidum, }\end{array}$

und andererseits

$$
\begin{array}{ll}
\text { hơfud } & \text { pogul-l } \\
\text { họf } \partial \mathrm{i}, \text { st. * hafuði } & \text { pọgl-ir, st. pagulir? }
\end{array}
$$

Dass dies für die priorität des $u$-umlautes spreche $\left.{ }^{2}\right)$, ist deshalb nicht anzunehmen, weil das späte (s. oben p. 147) $u$ in ul (z. b. in bogull) diesen umlaut im Westnordischen auch bewirkt, während er im Schwedischen vor -ul gänzlich fehlt. So war denn auch das entgegengesetzte verhältnis soeben wenn auch nicht erwiesen, so doch wahrscheinlich gemacht. Es ist also die zweite mögliche erklärung vorzuziehen, dass nämlich die syncope des $i^{3}$ ) eher eintrat als die des $u$, was ja mit dem verhalten dieser vocale in der vocalischen declination völlig übereinstimmen wärde. (Vgl. Wimmer, Navn. p. 73 f.) Darnach wäre die reihenfolge in der lautentwicklung folgende gewesen:

$\begin{array}{llll}\text { "katilar } & \text { katil-r } & \text { ketill } & \text { ketill } \\ \text { "katila } & \text { katl-i } & \text { katli } & \text { katli } \\ \text { "katilam } & \text { katl-am } & \text { katlom } & \text { kọtlom. }\end{array}$

1) $i$ in tamiðr, taliðr, vaniðr, hulið, u. dgl. tällt auf. Ist hier wirklich $i$, vorwiegend in älteru hss., das alte $i=$ got. $i$ in nasips? Die rhaltung wäre dann eine ausnahme vom nordischen auslautsgesetze (vgl. oben p. 155), die um so auffallender wäre, da dies $i$ nur gerade nach kurzer stammsilbe erhalten wäre (vgl. auch oben p. 155 anm. 1). Ein -iðr (eigentlich - eðr, daher ohne umlaut) wäre in der dritten conjugation zu erwarten [habaips : hafidr = nimais : nemir = nimaina : nemi(n)]. Sollte also analogiebildung vorliegen? F'iirs 0stnordische vgl. Rydq. I, $431 \mathrm{fi}$.

2) indem nämlich dieser vor, der $i$-umlaut aber erst nach der syncope eingetreten wäre.

3) Es handelt sich hier zunächst nur um die ältere syncope des $i$ nach $\mathrm{k}$ urzem vocal. 
Das gleiche gilt von den participialformen lagiðr, logð, lagit, wie man auch über das alter des $i$ und thber seine entstehung denken möge, also:

$\begin{array}{llll}* \text { lagiðar(?) } & \text { lage } \delta-r(?) & \text { lageðr } & \text { lagiðr } \\ \text { "lagiða(?) } & \text { lage }-u(?) & \text { lagðu } & \text { logð }\end{array}$

Ueber lage $\partial r$ siehe s. 161, anm. 1.

Die syucope des $i$ witrde also dem eintritt des stamm-iumlautes vorangegangen, dic syucope des $u$ dem stamm- $u-u m-$ laut nachgefolgt sein. Die reihenfolge wäre also diese: syucope des $i$, stamm- $i$-umlaut, stamm-u-umlaut, syncope des $u$, flexions-u-umlaut (sowol durch ursprungliches als auch durch unursprïngliches $u$ : toldum, log $\delta$, kotlom). Letztere aufeinanderfolge ergibt sich daraus, dass die syncope des $u$ (mit wenigen ausnahmen im Altschwedischen, s. Rydq. II, 402) gemeinnordisch, der flexious-u-umlaut aber (mit wenigen ausnahmen) nur westnordisch ist. - Nach dem hier angenommenen verbältnisse des stamm- $i$ - und $u$-umlautes wird doch auch wol folgende parallelentivicklung anzusetzen sein:

$$
\begin{aligned}
& \text { sinkvan } \left.<\text { sekkva }{ }^{1}\right)<\text { sökkva } \\
& \text { sankvjan }<\text { sekkva }<\text { sökkva. }
\end{aligned}
$$

Dass hier der umlaut des $j$ der ältere ist (gegen Holtzmanns annahme, oben p. 14ī), dafür spricht das völlig ${ }^{2}$ ) mangelude $j$, und das noch vorhandene $v$; ferner die noch vorhandene ubergangsstufe mit $e$ neben $\ddot{o}$, z. b. gerva neben görva, wälrend ich ein gorva nicht nachzuweisen wiiste; altschwed. engd neben isl. öngd, u. a. m. [vgl. Leffler, v-uml. 12-14]. Mehrfach ist $v$ von urspr. $v j$ im Nordischen vor dem umlaut ausgefallen, so in benda > bandja, got. bandvjan; dengja (ostnord. diunga, Leffler, v-uml. 67); öfter im Ostnordischen, so in sänkia u. lgl..

1) ink $<e k k$. Der übergang von $i<e$ steht wol mit der nasalierung und dem verklingen des nasals in zusammenhang (vgl. dri(g)kan $<$ diekka, $u(n)$ sis < oss, u(g)kara < okkar, "pu(n)hta (oder pullta?) $<$ pohta < potta; vgl. brekka und brink, klettr und klint u. a., s. Blomberg 3.1. Leffler (v-uml. 93 f.) bält enk, eny für die nordische grundform, nicht ink, ing, also seonka iilter als siunka, aber gleichzeitig mit beorn. Ich kann mich dieser aufiassung nicht anschliessen, indem ich auch $i v$ in biorn für $u$-umlant von $i a$ halte.

2) Erhalten ist es nur nach guttural, woflir Winner $(\$ 1+3$, c) zu vergleichen ist. 
vgl. 145 anm. 2). Mehrfach verschmolz es mit a zu au, so in ${ }^{*}$ davja < deyja, perf. do statt dov; in *havi < hey neben $*$ ha-gg-v-ja $<*$ heggva $<$ höggva.

Ueber den ausfall des $v$, dass $v$ nämlich riemlich spät abfiel, unterrichtet uns die verbindung $-h v$-. Hier muss (wie auch sonst im inlaut) $h$ verhältnismässig früh ausgefallen sein, nämlich friiher als $v$, denn $h$ zeigt sich nirgends mehr, während $v$ nicht selteu noch erscheint und auch noch umlaut wirkt, z. b. in ör-v-, got. arhwa-zna; ó neben á, got. ahva; fiör-v-, got. fairhvus: $v$ fällt also fort nach dem ausfall des $h$, und nach dem stamm- $u$-umlaut. Daher sind die von Leffler, $i$-uml. 12 augesetzten übergangsformeu * sehir, * léha u. dgl. unmöglich. Dass $h$ früler ausfiel als $v$, lässt sich auch auf folgende weise zeigen:

$$
\begin{aligned}
& * f e h-u<f e ́ \\
& * \text { feh-ar }<\text { féar }<\text { fjár } \\
& \because \text { feh-am }<\text { féam }<\text { fjám durch 'umspringen der quan- }
\end{aligned}
$$

titï' (Wimmer $\$ 20)^{1}$ ). feham ist fruhe analogiebildung statt des vereinzelten * /eh-um. Junger dagegen ist ler ausfall des $v$ in:

$$
\begin{aligned}
& \text { trev-(a) }<\text { tré, } \\
& \left\{\begin{array}{l}
\text { trev-am }<\text { tréam }<\text { trjám } \\
\text { trev-on }<\text { tréom }<\text { trjóm, }
\end{array}\right.
\end{aligned}
$$

ebenso knjäm und knjóm. Hiermit gleich steht

$$
\begin{aligned}
& \text { se(h)va(n) }<\text { sé(v)a }<\text { séa }<\text { sjá, } \\
& \text { se(h)va }<\text { sé(v)i }<\text { séi }<\text { sé, ébenso sér, }
\end{aligned}
$$

1) Das ist anschoinend nur ein westnordisches lautgesets: altschwedisch fea, trea (1l. i. féa, tréa), fear (und feiar, auch isländisch, übergangsform?), knam und tr('m neben knaram, tracem (dann auch wie allgemein mit om > am: tr(tom, kh(com); sc aus sía (isl. sjá) und seem, d. i. sim aus siam > "se(h)vam (Rydly. I, 118; II, 135. 162) erklärt sich nur so. Nom. pl. firindr, altschwed: und neuschwed. fiender [altschwed. fiander u. dgl. sind analogiebildungen nach dem gen. und dat.| ist wol so zu erklären, dass fiendr die gemeinnordische form, fjándr aber eine westnordische analogiebildung nach dem sing. fjändi ist. Allerdings erleidet $j i$ anscheinend iiberhaupt keinen unlaut; doch ist der $i$-umlaut in consonantischen stïmmen wol ziemlich alt und jedentalls gemeinnordisch. Ist also das umspringen der quantität nur westnordisch, so muss fiendr die gemeinnordische form sein. (Nach Gislason, Aarb. $186 t^{2} .295$ f.' wäre $i a ́$ hier überhaupt zweifelhatt.) 


$$
\left\{\begin{array}{l}
\text { se(h)vam } \left.<\text { sé(v)am }<\text { séam }<\text { sjám }{ }^{1}\right), \\
\text { ise(h)vom }<\text { sé(v)om }<\text { séom }<\text { sjóm, } \\
\text { trevo (nom. } p(.) \quad<\text { tréo }<\text { trjó } \\
\text { tre(v) }
\end{array}\right.
$$

ebenso kné und knjó. Dass diese formen in der tat so aufzufassen sind und dass nicht, wie Schmidt (Vocal. II, 409) will, anzusetzen ist:

$$
\begin{aligned}
& \text { treovam }<\text { treavam }<\text { treaam }<\text { treám, } \\
& \text { feoham }(\text { ?) }<\text { feaham }<\text { feaam }<\text { feám, }
\end{aligned}
$$

und ebensowenig trí aus $\left.\operatorname{treov}(a)^{2}\right)$, fí aus feoh(u), das folgt daraus, dass 1) dann die brechung lange vor dem fortfall des flexionsvocals stattgefunden haben müste, was nach allen andern beobachtungen schwerlich möglich ist; 2) dass die zweisilbigen formen auf $j a ́$ sich nur da zeigen, wo wirklich ein endungsvocal $a$ oder $o$ noch in nicht allzu fernliegender zeit vorhanden war, nämlich gen. sing. $-a r$, nom. acc. pl. ntr. - $[u, o]$, gen. pl. -a, dat. pl. -am, om; nicht aber, wo dies nicht der fall war: nom. sg. knev $(a)<k n i$, nicht knjá, knjó; gen. sg. *knevs < knés, nicht knjás * se(h)vir < sér, nicht sjär, was doch der fall sein muste, wenn der zweilaut durch brechung entstanden, also stammhaft wäre. Die nebenformen auf $j a ́$ treten eben nur da hervor, wo in nicht fernliegender zeit $a$, die nebenformen auf $j o ́$, wo $o$ in der endung stand. - Aehnlich liegt die sache bei klé und lé statt *kléi und léi; gen. kljá, lja statt*kléa, *léa; nom. pl. kljár, ljär statt*kléar, *léar; dat. pl. kljäm, ljám statt * kléam * léam. Wahrscheinlich ist hier $v$ ausgefallen (Schmidt a. a. 0. p. 409), also stände kléam statt *klé(v)am, léam statt * lé(v)am.

Aus diesen ausfuhrungen scheint mir hervorzugehen:

1) dass, als $v$ im inlaute ausfiel, das $o(u)$ im nom. acc. pl. ntr., also doch auch wol im nom. sing. fem., noch vorhanden, aber schon im schwinden begriffen war, daher kni neben knjó, dass also hier der übergang des ursprunglichen " zu $o$ fur die genannten casus schon in der vergangenheit lag, während er im dat. pl. und in der 1. pers. pl. des zeitworts eben erst begann, daher trjám neben trjöm, sjám neben sjóm

1) Formen wie sjium sind jïngere analogiebildungen, s. Wimmer S 107, anm. 1 .

$\left.{ }^{2}\right)$ Die formen auf $j o$ hat Schmidt nicht beriicksichtigt. 
u. s. w. - Also der iibergang des $a<u$, o trat im ersten falle früher ein als im zweiten.

2) dass, als $h$ in inlaute ausfiel, um noch nicht im iibergalige zu om hegriffen war, daher nur feam, fjäm; dass aber der stanmin-auslaut " abgefallen war, daher fí, nicht fjí, fjó.

Ein dritter derartiger ausfall ist der des $s$ zwischen vocalen in:

$$
\begin{aligned}
& \text { járn } \left.>i u r n>i s a r n, \text { noch mehrfach belegt }{ }^{1}\right) ; \\
& \text { vár }>\dot{a} a r>\text { isar }>\text { nnsara. }{ }^{2} \text { ) }
\end{aligned}
$$

Ehenso Bugge ['Tidskr. f. Phil. ogr Paed. XI, $121 \mathrm{f}$, wo er auch grjá aus * grisa und sjá (neben sá, sú) aus * sesá erklärt]. Damit, dass hier das verklingren des nasals bei nasalierung des vorhergehenden vocals den ausfall des $s$ voraufgegangen scin muss, stimmt es überein, dass das verklingen des nasals gemeinnordisch sein muss; doch war die nasalierung noch um 1150 auf Island hörbar (s. Pórodds traktat in der Edda), z. b. unterschied man in der aussprache $i>$ in, eri $>$ yngri, foer $>{ }^{*}$ fangir von andern gleichgeschriebenen wörtern (vgl. Lyngby, 'lidskr. f. Phil. og Paed. II, 317). Das verklingen des $n$ wird also wol nicht viel vor der nordischen sprachspaltung begonnen haben, vielleicht gleichzeitig mit dem verklingen des auslautenden $n$ in $n s$, welches ja erst nach abfall des auslautenden $r$, urspringlich $s$, zugleich mit dem ur-. sprünglich auslautenden $n$ verklingen konnte, wie denn auch eine inschrift im ältern runenalphabet (der Tune-stein, s. Wimmer, Navn. p. $44 \mathrm{f}$.) ein solches $n$ [in daedun? (dalidun? dallidun? Bugge, 'Tidskr. VIII, 191) und gahalaiban] noch zweimal bewahrt zeigt.

1) Erhalten ist $s$ in isarn noch hei den Skalden des IX. und X. jahrh. (denn an der ächtheit der ihnen zugeschriebenen lieder und strophen zu zweitelı, sehe ich in (len meisten fällen keinen grund), vgl. auch die bemerkung in Sn. E. II, 26 iiber éarn-hringar (urspr. wol iarn-) bei $O^{\prime}$ ttarr svarti (anfang des XI. jahrh.).

2) Vgl. aber or- (alt auch or- init längezeichen), die älteste form des possessiv, C.-V. 686. Wird also unsara < ${ }^{*}$ osar (vgl. p. 162 anm. 1)

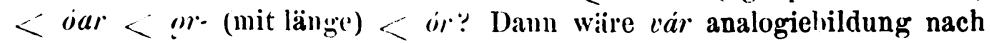
vèr (vgl. kvámum neben kómum u. igl.).
LEIPZIG, im Mär'z 1877.
A. EDZARDI. 